\title{
DESCRIPCIÓN ANESTESIOLÓGICA DEL EFECTO DE UN PROTOCOLO EN BASE A AZAPERONA Y KETAMINA EN CONEJO DOMÉSTICO (ORYCTOLAGUS CUNICULUS)
}

\author{
ANESTHETIC DESCRIPTION PROTOCOL BASED IN AZAPERONE \\ AND KETAMINE IN DOMESTIC RABBIT (ORYCTOLAGUS CUNICULUS)
}

\author{
Estefanía Flores P. (M.V.), Paulina Caroca E. ${ }^{1}$ (M.V.), Gino Cattaneo U. ${ }^{1}$ (M.V.), \\ Andrés Bastías P. ${ }^{1}$ (M.V.), AngéliCA Morales M. ${ }^{1}$ (M.V., M.S.)
}

\begin{abstract}
The effects of the anaesthetic protocol, based on a premedication with atropine and azaperone (Stresnil $\mathbb{R})$ and the anesthetic induction with a $0.5 \%$ ketamine solution, were studied in 25 healthy adults rabbits.

Each animal was premed with $0.04 \mathrm{mg} / \mathrm{kg}$ b.w. atropine, and $1.5 \mathrm{mg} / \mathrm{kg}$ azaperone to provide relaxation and decrease the muscle hyper-tonus produced by the dissociative anesthetic. After each drug administration, the physiologic parameter measure was registered.

The ketamine induction was done with a $0.5 \%$ ketamine solution, and the induction was determined by the lost of both, the straight head and the righting reflex. The heart rate, respiratory rate and arterial blood pressure were determined and registered every 5 minutes, the body temperature was controlled every 15 minutes, every measurement was done until the moment of recovery of the both previously mentioned reflex. The average dose of ketamine injected to achieve the induction anesthetic state, the time it takes the administration of that ketamine dose, the resultant surgical anesthetic period in minutes, and the post anesthetic motor depression time, were also determined and recorded.

The average I.V. dose induction of 0,5\% ketamine solution was $4.49 \mathrm{mg} / \mathrm{kg}$ of body weigh, injected in a average time of 9.56 seconds, providing a surgical anesthetic period of 10.64 minutes followed by a post anesthetic motor depression period of 5.64 minutes.

Under the effects of the anesthetics drugs association conforming this protocol, almost all physiological parameters remains in the normal ranges described for the specie, only the arterial blood pressure exhibited an average value under the normal value given for rabbits in the literature.
\end{abstract}

KEY WORDS: Anesthesiology,rabbit.

Palabras ClaVe: Anestesiología, conejo.

\section{INTRODUCCIÓN}

El conejo doméstico (Oryctolagus cuniculus) ha sido utilizado históricamente como animal de producción de pelo y carne, además de modelo animal para realizar una gran variedad de procedimientos experimentales, con fines de investigación, docencia y de adiestramiento quirúrgico (Luo et al., 1995). Sin embargo, el interés por tenerlo como animal de

Facultad de Ciencias Veterinarias y Pecuarias, Universidad de Chile. Casilla 2, Correo 15, La Granja, Santiago, Chile. compañía ha llevado a un aumento en las atenciones clínicas en esta especie, y a la necesidad de realizar una serie de procedimientos diagnósticos o terapéuticos que requieren de anestesia general (Huerkamp, 1995). El conejo doméstico presenta características especiales que lo convierten en un individuo susceptible a sufrir complicaciones durante la anestesia general. Su centro respiratorio es muy sensible frente a los agentes anestésicos (Aeschbacher, 1995). Su caja torácica es muy flexible, característica de adaptación de los animales de madriguera, lo que hace que la capacidad pulmonar residual se aproxime a la capacidad vital. La frecuencia respiratoria en reposo es 
alta (60 ciclos por minuto) y bajo anestesia profunda la respiración es lenta y con poco movimiento de la caja torácica lo que predispone a la atelectasia e inadecuada oxigenación, por lo que el tiempo que estos animales pueden ser mantenidos bajo anestesia general profunda es limitado (Sedgwick, 1988). Su pequeño tamaño determina una gran superficie corporal de contacto con el medio ambiente, lo que facilita la pérdida de calor corporal por radiación (Huerkamp, 1995). Estas características de la especie obligan a la necesidad de contar con información específica acerca de la respuesta a determinadas asociaciones de fármacos anestésicos, y de esta manera elaborar protocolos anestésicos seguros para su utilización en conejos de laboratorios y en aquellos que son pacientes en la práctica profesional.

Entre los agentes anestésicos más utilizados en medicina veterinaria está la ketamina, por sus características de corta acción, excelente soporte cardiovascular y mínimo efecto respiratorio, comparado con otros anestésicos inyectables. Ha sido usada en especies muy excitables como son los animales silvestres, siendo por esto recomendable para el conejo. Entre sus efectos, produce hipertono muscular, condición indeseable en procedimientos quirúrgicos que requieren de una buena relajación muscular. Esto se puede prevenir mediante su asociación con fármacos que provean una buena relajación muscular, como la azaperona, tranquilizante perteneciente a la familia de las butirofenonas, grupo de fármacos que comparten muchas características farmacológicas con las fenotiacinas, como los efectos tranquilizante y antiemético, siendo menos marcado su efecto hipotensor, no obstante producen más signos extrapiramidales, por lo que apenas se usan en clínica de pequeños animales (Laredo et al., 2001). La azaperona se considera bastante útil como tranquilizante de corta duración, por ejemplo en el transporte de animales y otra ventaja respecto de los derivados fenotiazínicos es que interfiere mínimamente con la termorregulación (Read M., 2002). Se ha usado en cerdos, en equinos y en la inmovilización y sedación de animales silvestres (Flores y Cattaneo, 2000). Su administración puede causar tremores, catalepsia y cambios de comportamiento (Mandsager y Raffe, 1989).

Los agentes anestésicos disociativos, como la ketamina, producen gran salivación, haciendo de uso casi obligado a la atropina, fármaco anticolinérgico ampliamente utilizado en medicina veterinaria, que disminuye la secreción de las glándulas salivales, contrarrestando la hipersalivación provocada por los disociativos como la ketamina y tiletamina (Hillyer y Quesenberry, 1997). La atropina facilita la intubación traqueal al mantener limpia y bien visible la zona laríngea y permite además estabilizar la frecuencia cardiaca, contrarrestando la bradicardia vagal originada por los fármacos vagotónicos, la intubación traqueal, la presión ocular y la tracción visceral (Flores y Cattaneo, 2000) .

Esta investigación tuvo por objeto describir los efectos anestesiológicos de un protocolo anestésico en base a la asociación de atropina, azaperona y ketamina, en el conejo doméstico (Oryctolagus cuniculus).

\section{MATERIALES Y MÉTODOS}

\section{Material Biológico}

Se utilizaron 25 conejos de 1 año de edad, clínicamente sanos, machos y hembras, de un peso corporal de 2,55 $\pm 0,7$ kilos.

\section{Material Anestesiológico}

El protocolo en ensayo incluyó: Sulfato de atropina, ampollas 1ml (1/ 1000); Azaperona, frasco ampolla de $50 \mathrm{ml}$ de solución (4\%); Ketamina, frasco ampolla de $10 \mathrm{ml}$ de solución (10\%); ampollas de agua bidestilada y suero fisiológico. Para su administración se utilizó: equipo de infusión scalp vein de $23 \mathrm{G}$ y $25 \mathrm{G}$; jeringas desechables de 1 y $10 \mathrm{ml}$. Los equipos de monitoreo usados fueron: Fonendoscopio Marshall; Termómetro Digital Philips; medidor de presión arterial ultrasónico doppler transcutáneo U.F. Model Bol-b Park Médica Electrónico D, y los procedimientos, variables y efectos del protocolo anestésico se registraron en una ficha de anestesia.

\section{MÉTODOS}

\section{Procedimiento experimental y registro de las variables fisiológicas}

Los conejos en ayuno de 12 horas fueron sometidos a un examen físico, determinando la ausencia de patologías evidentes. Se registró en la ficha de anestesia el peso, sexo, estado físico y las variables fisiológicas al T0: frecuencia cardiaca, frecuencia respiratoria y temperatura. Luego se administró sulfato de atropina en dosis de $0,04 \mathrm{mg} / \mathrm{kg}$ de peso corporal, vía intramuscular (IM), y a los 5, 10 y 15 minutos posteriores a la aplicación de atropina se midieron las 
frecuencias, cardiaca y respiratoria, y la temperatura a los 15 minutos, registrando los valores obtenidos. A continuación se administró azaperona en dosis de $1,5 \mathrm{mg} / \mathrm{kg}$ de peso corporal vía intramuscular, realizando a los $5,10,15$ y 20 minutos siguientes la medición de las frecuencias, cardiaca y respiratoria, y la temperatura a los 15 minutos de la administración de azaperona, registrando los valores obtenidos. Se canuló la vena marginal de la oreja, con un equipo de infusión scalp vein $25 \mathrm{G}$ o $23 \mathrm{G}$ y se conectó al paso de suero fisiológico, a una velocidad de $10 \mathrm{ml} / \mathrm{kg} / \mathrm{hr}$ y se procedió a la inducción lenta con ketamina al $0,5 \%$, hasta alcanzar el nivel de inducción anestésica, anotando la dosis del anestésico administrada y el tiempo necesario para realizar la inducción. Se instaló el Doppler transcutáneo y el ensayo continuó con el control de las variables fisiológicas antes mencionadas, incluyendo presión arterial sistólica, cada 5 minutos, y de la $\mathrm{T}^{\circ}$ cada 15 minutos, hasta que el individuo alcanzó una completa recuperación motora. Se midieron y anotaron los tiempos correspondientes a las variables anestesiológicas.

\section{Determinación de las variables anestesiológicas}

Dosis de inducción anestésica endovenosa (D.I.A.): miligramos de ketamina por kilogramo de peso corporal del conejo, que administrados por la vía endovenosa, permiten alcanzar un nivel de anestesia general definido por el momento en que el individuo pierde el reflejo de cabeza erguida y estación.

Tiempo de inducción anestésica (T.I.A.): periodo de tiempo en segundos, que se inicia con la administración endovenosa del fármaco y que termina cuando se alcanza el nivel anestésico antes indicado.

Tiempo de anestesia quirúrgica (T.A.Q.): Periodo de tiempo, en minutos, medido desde la inducción hasta la recuperación de la sensibilidad, que se determina por la presencia de respuesta motora, frente al estímulo producido al pinzar un pedazo de piel con una pinza hemostática en las orejas, en el cuello y la zona lumbar. Este tiempo es importante, ya que permite estimar qué procedimientos es posible realizar con sólo administrar la dosis de inducción.

Tiempo de depresión motora postanestésica (T.D.M.P.A.): Periodo de tiempo que va desde la recuperación de la sensibilidad, hasta la recuperación motora voluntaria, manifestada por la presencia del reflejo de cabeza erguida y de estación. La importancia de esta variable es que la recuperación de la actividad motora incluye los mecanismos protectores que contribuyen a la conservación de la vida del individuo.

Tiempo de anestesia total (T.A.T.): corresponde a la sumatoria del tiempo de anestesia quirúrgica con el tiempo de depresión motora postanestésica.

\section{Método Estadístico}

Con los datos registrados se calcularon el Promedio, la Desviación estándar y el Coeficiente de variación de las variables anestesiológicas, para dar cuenta de las características particulares de las muestras poblacionales obtenidas. Además se realizó un análisis de varianza entre las constantes fisiológicas registradas a fin de determinar la existencias de diferencias significativas en el tiempo y una prueba de comparaciones múltiples de Tukey-Kramer, para identificar en qué tiempos se produjeron estas diferencias. Para establecer las relaciones que pudieran existir entre las variables anestesiológicas medidas, se calculó el coeficiente de correlación de Pearson.

\section{Efectos colaterales}

Se registró y describió su presentación, asociados al protocolo anestésico.

\section{RESULTADOS Y DISCUSIÓN}

\section{Determinación de las variables anestesiológicas (Tabla 1)}

La dosis de inducción anestésica para el conejo doméstico fue de $4,49 \pm 0,93 \mathrm{mg} / \mathrm{kg}$, con un tiempo requerido para la inducción de 9,56 $\pm 2,63$ segundos, logrando una anestesia quirúrgica por 10,64 $\pm 3,12$ minutos, un tiempo de depresión motora de 5,64 \pm 1,49 minutos y un tiempo total de anestesia de 16,28 $\pm 2,9$ minutos. De las relaciones entre las variables anestesiológicas, es importante considerar que a mayor dosis de inducción anestésica mayor es el tiempo depresión motora posterior a la anestesia, que demandará un periodo de cuidado intensivo más prolongado (Tabla 2).

\section{Efecto del protocolo anestésico sobre las variables fisiológicas \\ - Frecuencia Cardiaca (Figura 1)}

La frecuencia cardiaca basal promedio (T0) fue de $246,7 \pm 31,4$ latidos/minuto. Posterior a la administración de atropina no se observan variaciones significativas, lo que concuerda con lo descrito para la especie por diversos autores como Flores y Cattaneo, 
TABLA 1

DESCRIPCIÓN ESTADÍSTICA DE LAS VARIABLES ANESTESIOLÓGICAS

EN CONEJOS BAJO EL PROTOCOLO ATROPINA - AZAPERONA - KETAMINA

\begin{tabular}{l|c|c|c|c|c}
\hline & $\begin{array}{c}\text { DIA } \\
(\mathrm{mg})\end{array}$ & $\begin{array}{c}\text { TIA } \\
\text { (segundos) }\end{array}$ & $\begin{array}{c}\text { TAQ } \\
\text { (minutos) }\end{array}$ & $\begin{array}{c}\text { TDMPA } \\
\text { (minutos) }\end{array}$ & $\begin{array}{c}\text { TAT } \\
\text { (minutos) }\end{array}$ \\
\hline Promedio & 4,49 & 9,56 & 10,64 & 5,64 & 16,28 \\
\hline D.E. & 0,93 & 2,63 & 3,12 & 1,49 & 2,90 \\
\hline C.V. (\%) & 20,74 & 27,52 & 29,33 & 26,53 & 17,86 \\
\hline Mínimo & 2,5 & 5 & 6 & 2 & 11 \\
\hline Máximo & 6,6 & 14 & 19 & 8 & 24 \\
\hline
\end{tabular}

D.E.: Desviación estándar.

C.V.: Coeficiente de variación.

TABLA 2

RELACIONES ENTRE LAS VARIABLES ANESTESIOLÓGICAS

\begin{tabular}{l|c|c}
\hline Variables & $r$ & $P$ \\
\hline DIAT/PESO & 0,441517 & 0,027139 \\
\hline DIAT/TIA & 0,746160 & 0,000018 \\
\hline DIAK/TDMPA & 0,609456 & 0,001221 \\
\hline
\end{tabular}

Resultado del análisis de correlación de Pearson.

(2000) y Plunkett (2002) que indican la existencia de una enzima atropina esterasa que degrada el fármaco haciendo al conejo menos sensible a sus efectos.

Después de 20 minutos de la administración de azaperona se observa un descenso progresivo de la frecuencia cardiaca llegando a los $205,7 \pm 32,8$ latidos/minuto, variación que fue significativa con respecto a la frecuencia cardiaca basal y a la medida bajo efecto de atropina. Esto corroboraría el efecto bardicardizante de la azaperona, descrito en cerdos por el laboratorio Norvet (2005), el cual recomienda este fármaco para regular los procesos de sobrecarga cardiaca en esta especie.

A los 5 minutos de administrada la ketamina la frecuencia cardiaca se eleva desde 205 a 225 latidos por minuto, sin embargo este aumento no es estadísticamente significativo, además, en los registros posteriores se observó una disminución de esta frecuencia hasta 201 latidos por minuto al final del ensayo, siendo éste estadísticamente significativo. Este comportamiento sería lo esperado según lo descrito por Flores y Cattaneo (2001), ya que la ketamina brinda un excelente soporte cardiovascular y, por lo tanto, es capaz de contrarrestar los efectos depresores hemodinámicos de la azaperona. Es im-

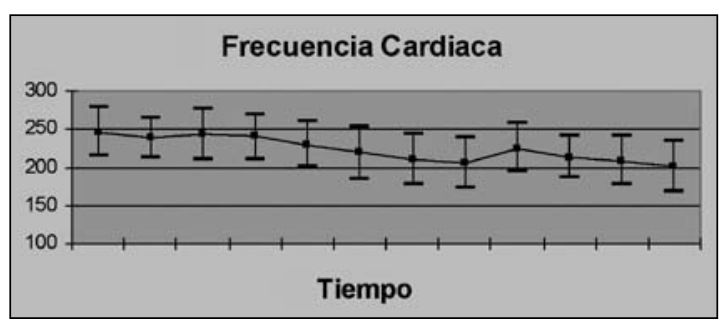

Figura 1. Variación de la frecuencia cardiaca en el tiempo (promedio \pm desviación estándar) en conejos bajo el protocolo anestésico.

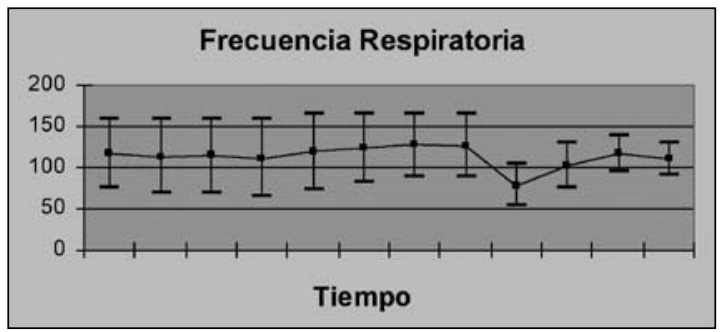

Figura 2. Variación de la frecuencia respiratoria en el tiempo (promedio \pm desviación estándar) en conejos bajo el protocolo anestésico.

portante mencionar que todos los promedios de la frecuencia cardiaca registrados se encuentran dentro de los rangos normales descritos para la especie por Hillyer y Quesenberry (1997), lo que demuestra la seguridad desde el punto de vista cardiovascular del protocolo estudiado.

\section{- Frecuencia Respiratoria (Figura 2)}

La frecuencia respiratoria basal promedio de 117,4 $\pm 40,9$ respiraciones/minuto, luego de aplicada la atropina, si bien presentó un descenso en el promedio registrado, este fue leve y no significativo, llegando a los 15 minutos de administrada la atropina a las $111,8 \pm 45,8$ respiraciones/minuto. 
Al administrar azaperona se observa un aumento no significativo del promedio, alcanzando su máximo a los 15 minutos con 127,2 $\pm 38,5$ respiraciones $/ \mathrm{mi}$ nuto, lo que concuerda con el laboratorio Norvet que describe una posible presencia de taquipnea durante su utilización y bajo estados de sobredosis, luego de lo cual hay un descenso brusco registrado a los 5 minutos de administrada la ketamina, en que el promedio llega a 79,2 $\pm 25,2$ respiraciones/minuto, valor mínimo alcanzado durante el ensayo y que representa una diferencia estadísticamente significativa, pero luego aumenta hasta el minuto 15 llegando a $117,2 \pm 22,5$ respiraciones/minuto, valor semejante al promedio basal, que sufre una leve disminución. Esto concuerda con Thurmon et al., 1999 que describe una respiración apnéustica tras la aplicación de ketamina, que lleva a una disminución de la frecuencia respiratoria, pero al no verse afectada la respuesta ventilatoria a la hipoxia, esta se regulariza prontamente. Es importante señalar que en este estudio la frecuencia respiratoria inicial estuvo por sobre lo normal para la especie, lo que puede ser atribuido al estrés de la manipulación. Además es importante destacar que los valores de la frecuencia respiratoria nunca disminuyeron a valores subnormales durante el transcurso del ensayo.

Al igual como ocurrió con la frecuencia cardiaca, la frecuencia respiratoria también muestra una diferencia importante luego de 15 minutos de administrada la azaperona, aunque significativa sólo en una de ellas, lo que concuerda con el periodo de latencia del fármaco administrado por vía i.m., descrito por Flores y Cattaneo (2000).

\section{- Presión arterial sistólica (Figura 3)}

Para una colocación eficiente del doppler transcutáneo en la zona palmar del miembro anterior, la presión arterial sistólica se midió desde la inducción anestésica hasta el fin del periodo de depresión motora postanestésica. El valor promedio inicial fue de $63,8 \pm 12,3 \mathrm{~mm} \mathrm{Hg}$, no presentándose cambios significativos en el promedio durante el ensayo. Es importante considerar este valor, ya que se encuentra por debajo de la mínima indicada para la especie por Haskins, 1995 que la sitúa en $80 \mathrm{~mm} \mathrm{Hg}$. En cambio, en trabajos realizados por Lipman, 1990 indican una presión arterial promedio de $57 \mathrm{~mm} \mathrm{Hg}$ al usar la asociación atropina-xilacina-ketamina y de $46 \mathrm{~mm} \mathrm{Hg}$ al adicionar acepromacina al protocolo anterior. Los resultados obtenidos en esta experiencia concuerdan con Sedgwick, 1988; que indica que la ketamina en el conejo sólo induce un aumento de la

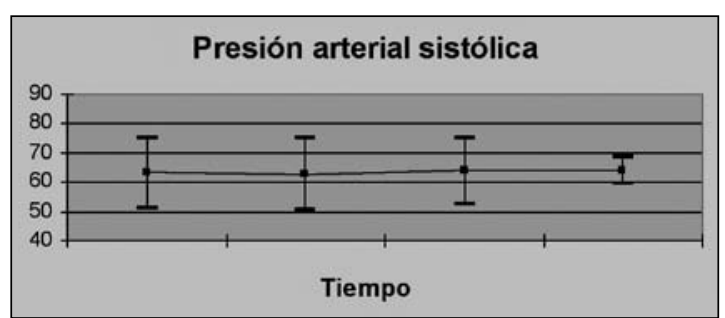

Figura 3. Variación de presión arterial sistémica en el tiempo (promedio \pm desviación estándar) en los conejos bajo el protocolo anestésico.

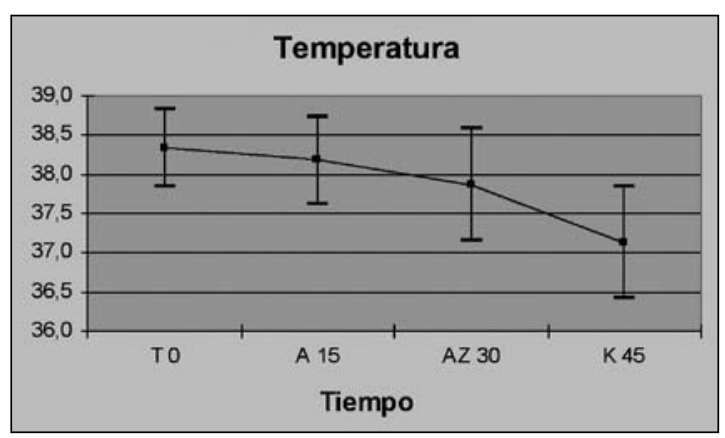

Figura 4. Variación de la temperatura en el tiempo (promedio \pm desviación estándar) en conejos bajo el protocolo anestésico.

presión sanguínea si se utiliza sola, ya que su efecto hipertensor no elimina la hipotensión que ocurre al mezclarla con tranquilizantes.

\section{- Temperatura (Figura 4)}

El promedio de la temperatura basal de $38,3 \pm 0,48{ }^{\circ} \mathrm{C}$, a medida que se desarrolla el ensayo experimentó un descenso sostenido, más evidente a los 15 minutos de administrada la azaperona, con un promedio de $37,8 \pm 0,70{ }^{\circ} \mathrm{C}$. A los 15 minutos de administrada la ketamina, se alcanza el valor mínimo de $37,1 \pm$ $0,71^{\circ} \mathrm{C}$, siendo ambas diferencias significativas.

\section{Efectos colaterales del protocolo anestésico}

(Tabla 3)

De los movimientos involuntarios evidenciados en el $32 \%$ de los conejos anestesiados, se diferencian 3 que exhibieron movimientos involuntarios de extremidades y 5 movimientos mandibulares rotatorios o masticatorios. Un 20\% de los individuos manifestó hiperestesia evidente al producir ruidos fuertes, pero no al estímulo doloroso. Cinco individuos (20\%) defecaron durante el desarrollo de la experiencia y uno orinó. También es importante señalar que en un $44 \%$ de los conejos machos se observó priapismo, reacción descrita en sobredosificaciones de azaperona en cerdos. 
TABLA 3

EFECTOS COLATERALES DEL PROTOCOLO ANESTÉSICO

\begin{tabular}{l|c|c}
\hline Efecto colateral & $N^{\circ}$ de animales afectados & Porcentaje \\
\hline Movimientos involuntarios & 8 & 32 \\
\hline Hiperestesia & 5 & 20 \\
\hline Micción & 1 & 4 \\
\hline Defecación & 5 & 20 \\
\hline Priapismo & $4 / 9$ & 44 \\
\hline
\end{tabular}

\section{RESUMEN}

Se estudiaron los efectos de un protocolo anestésico basado en una premedicación con atropina y azaperona (Stresnil@), e inducción con Ketamina preparada al $0,5 \%$ en 25 conejos adultos y sanos. Los animales fueron premedicados con atropina en una dosis de 0,04 $\mathrm{mg} / \mathrm{kg}$ para contrarrestar los efectos de predominio vagal derivados de la anestesia y azaperona en dosis de $1,5 \mathrm{mg} / \mathrm{kg}$, para proveer tranquilización y reducir el hipertono muscular que produce el anestésico, esperando reducir la hipotensión inducida por otros tranquilizantes como los fenotiacínicos. Posterior a la aplicación de cada uno de los fármacos se midieron las variables fisiológicas.

La inducción con ketamina se realizó hasta observar la pérdida del reflejo de cabeza erguida y estación. Se midieron cada 5 minutos la frecuencia cardiaca, frecuencia respiratoria y presión arterial, y cada 15 minutos la temperatura corporal, hasta la recuperación de los reflejos anteriormente descritos. Se registró además la cantidad de ketamina necesaria para la inducción, el tiempo empleado en la administración del fármaco, el tiempo de anestesia quirúrgica, y el tiempo de depresión motora pos anestésica.

En el conejo doméstico la dosis promedio de inducción endovenosa de la solución de ketamina al $0,5 \%$ fue de $4,49 \mathrm{mg} / \mathrm{kg}$, administrado en un tiempo promedio de 9,56 segundos, proporcionando un período de anestesia quirúrgica de 10,64 minutos y un período de depresión posanestésica de 5,64 minutos promedio.

Los cambios en las variables fisiológicas bajo la acción del protocolo estudiado se mantienen dentro de los rangos fisiológicos normales para la especie, excepto la presión arterial, la cual registra un promedio inferior al rango dado para la especie, pero superior a lo descrito en otros protocolos anestésicos en que no se utilizó la azaperona.

\section{REFERENCIAS}

AeschBaCher, G. 1995. Rabbit Anesthesia. Compendium Continuing Education of the Practicing Veterinarian 17:1004-1011.

FLORES, E.; CATTANEO, G. 2000. Técnicas anestésicas inyectables de uso actual. Premedicación y sedación. Monografías de Medicina Veterinaria. 20 (2): 34-48.

Flores, E.; CATTANEO, G. 2001. Técnicas anestésicas inyectables de uso actual II: Anestésicos inyectables. Monografías de Medicina Veterinaria. 21 (1): 40-54.

HASKINS, S.C. 1995. Anesthesic Management of patients with cardiopulmonary disorders. Congress of theWorld Veterinary Association 25; Yocohama, Japan. Pp. 190-197.

Hillyer, E.B.; QueSENBERRY, K.L. 1997. Ferrets, Rabbits, and Rodents Clinical Medicine and Surgery. W.B. Sounders Company. U.S.A. 432 pp.

HUERKAMP, M.J. 1995. Anesthesia and postoperative management of rabbits and pocket pets. Current Veterinary Therapy XII Small Animal Practice. 1322-1327.

LAREDO, F.; REDONDO, J.I.; GómEZ-VILlamandos, R.; BELDA, E.; CRUZ, J.I. 2001. La preanestesia: analgesia, inmovilización farmacológica, tranquilización y ansiolisis. Consulta Difusión Veterinaria 9 (77): 37-50.

LIPMAN, N; MARINI, R; ERDMAN, S. A comparison of ketamine/ xylazine and ketamine/xylazine/acepromazine anesthesia in the rabbit. Laboratory Animal Science. 1990 jul; 40(4):395-398.

LuO, Y.; Russell, G.; GRIFFITH, J.; LANG, M. 1995. Comparison of anesthesia induced by Ketamine-Fentanyl combination and maintained by Propofol or Etomidate in New Zealand White rabbits. Laboratory Animal Science 45 (3): 269-275.

MANDSAGER, R.; RAFFE, M. 1989. Chemical restraint techniques in dogs and cats. Current veterinary therapy X Small animal practice. 63-70.

PLUNKETT, S. 2002. Manual de urgencias en pequeños animales. 2th ed. Interamericana McGraw-Hill. México. 598 p.

SEDWICK, C. 1988. Anestesia para conejos y roedores, in: Terapéutica Veterinaria y Práctica clínica en especies pequeñas. México, Editorial Continental. 705-709. Thurmon, J.C.; TRANQUILLI, W.J.; BENSON, G.I. 1999. Essentials of Small Animal Anesthesia and Analgesia. Estados Unidos, Philadelphia Lippincott Williams e Wilkins. 580 p.

READ M. 2002. Long acting neuroleptic drugs. [en linea] In: Heard D.(Ed). Zoological restraint and anesthesia. <http://www.ivis. org/special_books/Heard/read/chapter_frm.asp?LA=1>

LABORATORIO NORVET S.A. 2005. Stresnil®, tranquilizante específico para cerdos. In: Vademécum. <http://www.norvet. es/vademecum.asp?id=148> [consulta 12-09-2005]. 